\title{
Dual-isotope myocardial perfusion SPECT imaging: Past, present, and future
}

\author{
Tali Sharir, MD, ${ }^{\mathrm{a}, \mathrm{b}}$ and Piotr Slomka, PhD ${ }^{\mathrm{c}, \mathrm{d}}$ \\ a Department of Nuclear Cardiology, Assuta Medical Center, Tel Aviv, Israel \\ b Faculty of Health Sciences, Ben Gurion University of the Negev, Beer Sheba, Israel \\ c Department of Imaging (Division of Nuclear Medicine) and Medicine, Cedars Sinai Medical \\ Center, Los Angeles, CA \\ d David Geffen School of Medicine at the UCLA, Los Angeles, CA
}

Received Jun 15, 2017; accepted Jun 15, 2017

doi: $10.1007 / \mathrm{s} 12350-017-0966-0$

\section{See related article, pp. 2016-2023}

Myocardial perfusion SPECT imaging (MPI) is a widely available technique used to identify and quantify ischemia, infarct, and myocardial viability. The first radioisotope used was thallium (Tl)-201, introduced to nuclear medicine by Kawana et al in $1970,{ }^{1}$ and evaluated for MPI shortly after. ${ }^{2}$ As a biologic analog of potassium, Tl-201 has the advantages of efficient myocardial extraction and rapid blood clearance, favoring its use as a myocardial perfusion agent. Accumulation of Tl-201 within ischemic zones is slower compared to non-ischemic regions, and reaches a peak after several hours. Thus, early post-Tl-201 injection images demonstrate flow, whereas delayed images demonstrate myocardial viability within the ischemic or infarcted zones.

Tc-99m-labeled tracers were introduced during the 1980s, and technetium (Tc)-99m sestamibi became commercially available in the United States in $1990 .{ }^{3}$ The shorter physical half-life of Tc-99m compared to Tl-201 (6 vs 73 hours) allowed the injection of a tenfold higher dose of 25 to $30 \mathrm{mCi}$ compared to $3 \mathrm{mCi}$, respectively. The higher injected activity resulted in higher myocardial count density and better image

Reprint requests: Tali Sharir, MD, Department of Nuclear Cardiology, Assuta Medical Center, 96 Igal Alon, C Building, 67891 Tel Aviv, Israel; tsharir@gmail.com

J Nucl Cardiol 2018;25:2024-8.

$1071-3581 / \$ 34.00$

Copyright (c) 2017 American Society of Nuclear Cardiology. quality compared to Tl-201 images, and allowed the acquisition of ECG-gated images of good quality. Moreover, lacking significant redistribution, Tc-labeled tracers offered flexibility of imaging protocols, using a 2-day stress and rest protocol, or 1-day either rest-stress or stress-rest protocols. Therefore, Tc-99m-labeled radiotracers, either sestamibi or tetrofosmin became dominant in nuclear cardiology.

During the early 1990s, dual-isotope MPI was first introduced by Berman et $\mathrm{al}^{4,5}$ using Tl-201 for rest, followed by Tc-99m sestamibi for stress imaging. This original protocol gained attention and popularity because it offered significant shortening of the entire test time from 4-5 hours to only 1.5-2 hours. In addition, it utilized advantages of both tracers for the assessment of myocardial ischemia and viability. The high count density of Tc-99m sestamibi provided good-quality gated SPECT stress images, allowing evaluation of stress perfusion deficit and post-stress left ventricular function, whereas resting Tl-201 provided assessment of resting perfusion, and the option to obtain delayed images for the assessment of myocardial viability within infarcted or severely ischemic regions. Ideally, acquisition of rest Tl-201 and stress Tc-99m should be obtained simultaneously in one SPECT acquisition. This would provide optimal image registration, save imaging time, and increase patient throughput. However, when using traditional sodium iodide ( $\mathrm{NaI}$ ) detectors and photomultipliers, the crosstalk of counts originating from the Tc-99m into the Tl-201 window is significant, and accounts for $27 \%$ of the counts acquired in the Tl-201 window. ${ }^{3}$ This is due to the limited energy resolution of the traditional Anger cameras. These "extra" counts may cause overestimation of ischemia and viability. Therefore, dual-isotope protocol has been used typically in the separate-acquisition mode. 
In recent years, the awareness to patient radiation exposure from MPI has increased, and the dual-isotope protocol has been criticized for exposing patients to the highest radiation level of all MPI protocols. ${ }^{6}$ As a result, the use of this protocol declined substantially. However, the introduction of new solid-state CZT cameras offered the opportunity to reduce radiation doses associated with all MPI protocols, and opened the horizon for reviving dual-isotope MPI. Moreover, using CZT detectors, simultaneous acquisition of Tc-99m and Tl-201 became feasible due to the higher energy resolution of these detectors compared to conventional cameras (at $140 \mathrm{keV} 5.5 \%$ vs $10 \%$, respectively). ${ }^{7}$

In the current issue of the journal, Imbert and colleagues from Nancy, France, evaluated a novel low-dose dual-isotope procedure using a CZT camera (D-SPECT, Spectrum Dynamics, Caesarea, Israel). Unlike the conventional separate-acquisition dual-isotope protocol which involved an initial resting Tl-201 injection and acquisition, followed by stress Tc-99m sestamibi injection and acquisition, ${ }^{4}$ the current protocol started with stress sestamibi injection and imaging followed by rest Tl-201 injection, and both Tc99m and Tl-201 acquired simultaneously 15 minutes after Tl-201 administration at rest. The study involved also a resting sestamibi injection and imaging which followed the dual-isotope imaging. Thus, stress Tc-99m-sestamibi/rest Tl-201 could be compared head to head with the conventional single-isotope stress/rest Tc-99m-sestamibi protocol. Processing of T1-201 images involved reduction in crosstalk from the Tc-99m window by analyzing information from multiple energy windows as previously described. ${ }^{7}$ Also, background upward shift of count level in the resting Tl-201 images was corrected by $12 \%$ to match background count level in the resting Tc-99m sestamibi images. The results demonstrated similar average resting uptake for background corrected Tl-201 and Tc-99m within ischemic or infarcted myocardial segments. Similar reversibility was obtained for singleisotope stress Tc-99m/dual-isotope rest Tl-201 compared to single-isotope stress Tc-99m/rest Tc-99m. ROC analysis to identify ischemic segments based on dualisotope stress Tc-99m and rest Tl-201 demonstrated similarly high diagnostic value for the dual and singleisotope techniques with area under the curve of $0.83 \mathrm{vs}$ 0.81 , respectively. The study demonstrated that both stress Tc-99m and rest Tl-201 obtained from simultaneous dual-isotope acquisition can be used for the assessment of myocardial ischemia.

The advantage of the protocol proposed by Imbert et al compared to previously described dual-isotope procedures is the involvement of stress-first Tc-99msestamibi with the optional conversion to a stress-only test in cases with a normal stress study, similar to the stress-first single-isotope Tc-99m procedure. As previously described, stress-only testing is an important measure for reducing patient radiation exposure in MPI. ${ }^{8}$ Figure 1 demonstrates effective radiation doses associated with the single- and dual-isotope procedures, based on injected activity doses in the current study by Imbert et al. A complete stress and rest dual-isotope testing still involved higher per-patient radiation dose compared to the single-isotope procedure ( $8.3 \mathrm{vs} 4.3 \mathrm{mSv}$, respectively) (Figure 1A), but accounted for much lower radiation dose compared to the traditional rest Tl-201/ stress Tc-99m sestamibi protocol using a conventional Anger camera (over $20 \mathrm{mSv}$ ), or previously reported dual-isotope protocols using a CZT camera. ${ }^{9-11}$ A stressonly rate of $40 \%$ would result in a substantial reduction of average effective radiation to $3 \mathrm{mSv}$ for the singleisotope and $5.4 \mathrm{mSv}$ for the dual-isotope procedure (Figure 1B).

Few studies evaluated the feasibility and diagnostic value of dual-isotope MPI using a CZT camera (Table 1). Ben-Haim et $\mathrm{al}^{9}$ demonstrated the feasibility and high image quality of simultaneously acquired dualisotope, rest Tl-201/stress Tc-99m-sestamibi MPI using a solid-state CZT camera, in comparison to conventional, separate-acquisition SPECT. The injected doses of Tl-201 and Tc-99m-sestamibi were 2.2 and $6.8 \mathrm{mCi}$, respectively, accounting for a total per-patient effective
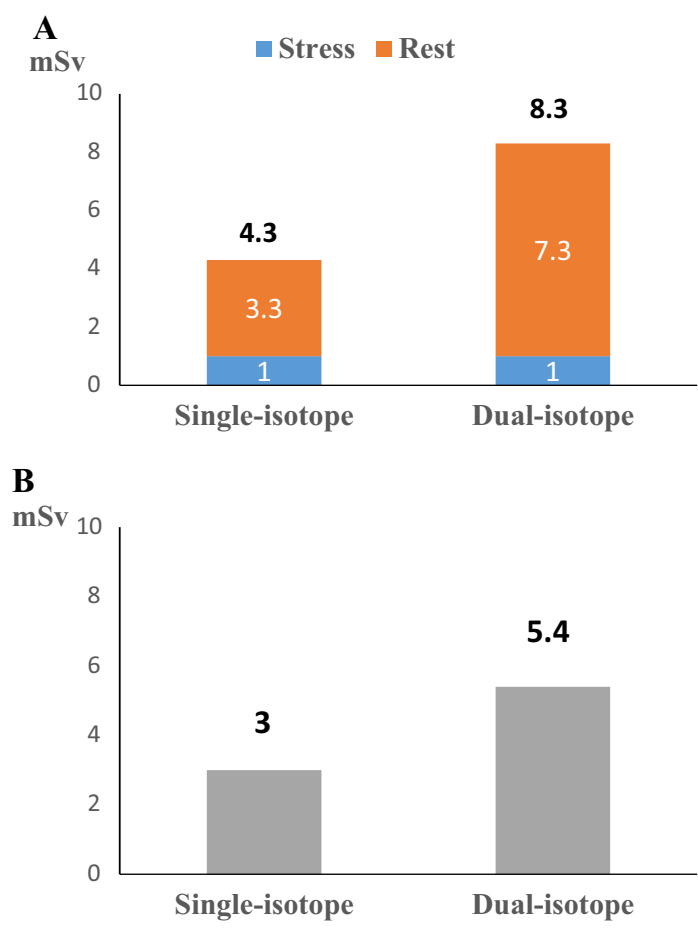

Figure 1. Effective radiation dose associated with reduced dose Tc-99m-labeled single-isotope and Tc-99m/Tl-201 CZTMPI. 
dose of $11.4 \mathrm{mSv}$. The simultaneously acquired data were corrected for spillover and scatter. ${ }^{7}$ The results of this study demonstrated comparable perfusion findings obtained by simultaneous rest Tl-201/stress Tc-99msestamibi CZT-MPI and separate, conventional dualisotope SPECT.

Berman et $\mathrm{al}^{10}$ offered a different version of the dual-isotope CZT-MPI, using separate acquisition of stress T1-201 and rest Tc-99m-sestamibi. They demonstrated similar image quality for this protocol compared to rest/stress single-isotope Tc-99m-sestamibi. This study also demonstrated the feasibility of early resting Tc-99m-sestamibi imaging, beginning within 2 minutes after rest injection, and suffering from less extra-cardiac activity than the conventional single-isotope protocol. Thus, the whole procedure was accomplished in $<20$ minutes. This protocol takes advantage of the higher extraction fraction and linearity of Tl-201 with coronary blood flow at high flow rates compared to Tcbased tracers $(0.85$ vs $\sim 0.60)$, with the potentially better detection of perfusion deficits appearing at high flow rates, and associated with mild coronary disease. The protocol involved effective radiation of $11 \mathrm{mSv}$ (8.7 mSv Tl-201/2.3 mSv Tc-99m-sestamibi) or $13.8 \mathrm{mSv} \quad(10.9 \mathrm{mSv}$ Tl-201/2.9 mSv Tc-99m sestamibi) for patients weighting $\leq 225$ and $>225 \mathrm{lbs}$, respectively.

Another interesting dual-isotope MPI protocol has been recently suggested by Makita and colleagues from Japan. $^{11}$ These investigators evaluated a CZT-MPI protocol, starting with rest Tc-99m-tetrofosmin injection, followed by adenosine stress Tl-201 and simultaneous acquisition of both isotopes. Patients also underwent a second dual-isotope imaging 30 minutes later for the comparison of ECG-gated wall motion with the immediately post-stress acquisition. Validation of
MPI results against invasive angiography defining obstructive coronary disease $\geq 75 \%$ stenosis yielded area under the ROC curve 0.908 on a per-patient basis, with sensitivity, specificity, and accuracy of $88.6 \%$, $79.2 \%$, and $86.2 \%$, respectively.

The current study by Imbert and colleagues demonstrates a dual-isotope protocol involving the lowest effective radiation of all dual-isotope protocols described to date (Table 1). Moreover, it is the only protocol that can be converted to a low-dose stress-only test upon a normal stress MPI, reducing effective dose from $8.3 \mathrm{mSv}$ for the complete stress and rest study to $1 \mathrm{mSv}$ stress-only testing. Other dual-isotope protocols either begin with resting injection, or start with stress Tl201 , which is associated with $\sim 8 \mathrm{mSv}$ radiation. Therefore, this protocol might have a more optimistic future compared to other dual-isotope protocols in this era, when reducing patient radiation exposure is crucial for the continuing use of SPECT imaging in nuclear cardiology. However, before implementing this protocol into clinical use it should be noted that it has not been validated against coronary angiography, and its clinical advantage in the detection of myocardial hibernation by additional late Tl-201 imaging has not been explored. To date, only one type of the commercially available CZT cameras, namely the D-SPECT camera, has been used in the published dual-isotope MPI studies, and methods for simultaneous acquisition of Tc-99m and Tl-201 with scatter and crosstalk corrections were developed for this specific camera. It is important that investigators develop and assess the utility of dual-isotope protocols using the Discovery 530c camera (GE Healthcare) as well.

The shift from Tl-201 to Tc-based tracers and the abandon of dual-isotope MPI procedures have rendered the field of SPECT-MPI without satisfactory measures

Table 1. Dual-isotope myocardial perfusion imaging protocols

\begin{tabular}{|c|c|c|c|c|c|c|c|}
\hline & $\begin{array}{c}\text { Camera } \\
\text { type }\end{array}$ & $\begin{array}{c}\text { First } \\
\text { injection }\end{array}$ & $\begin{array}{l}\text { Second } \\
\text { injection }\end{array}$ & $\begin{array}{l}\text { Stress } \\
\text { type }\end{array}$ & $\begin{array}{c}\text { Acquisition } \\
\text { mode }\end{array}$ & $\begin{array}{l}\text { Total } \\
\text { time }\end{array}$ & $\begin{array}{l}\text { Eff. } \\
\text { dose }\end{array}$ \\
\hline $\begin{array}{c}\text { Berman } \\
1994\end{array}$ & Conventional & Rest Tl-201 & Stress Tc-MIBI & Ex or $A D$ & Separate & 90 & 22 \\
\hline $\begin{array}{l}\text { Ben-Haim } \\
2010\end{array}$ & CZT & Rest Tl-201 & Stress Tc-MIBI & $A D$ & Simultaneous & 70 & 11.4 \\
\hline $\begin{array}{r}\text { Berman } \\
2008\end{array}$ & $\mathrm{CZT}$ & Stress Tl-201 & Rest Tc-MIBI & Ex or $A D$ & Separate & 20 & 11 \\
\hline $\begin{array}{l}\text { Makita } \\
2016\end{array}$ & $\mathrm{CZT}$ & Rest Tc-Tetro & Stress Tl-201 & $A D$ & Simultaneous & 70 & 10.6 \\
\hline Imbert 2017 & CZT & Stress Tc-MIBI & Rest Tl-201 & Ex or Dipy & Simultaneous & 75 & 8.3 \\
\hline
\end{tabular}

$E x$, exercise; $A D$, adenosine 


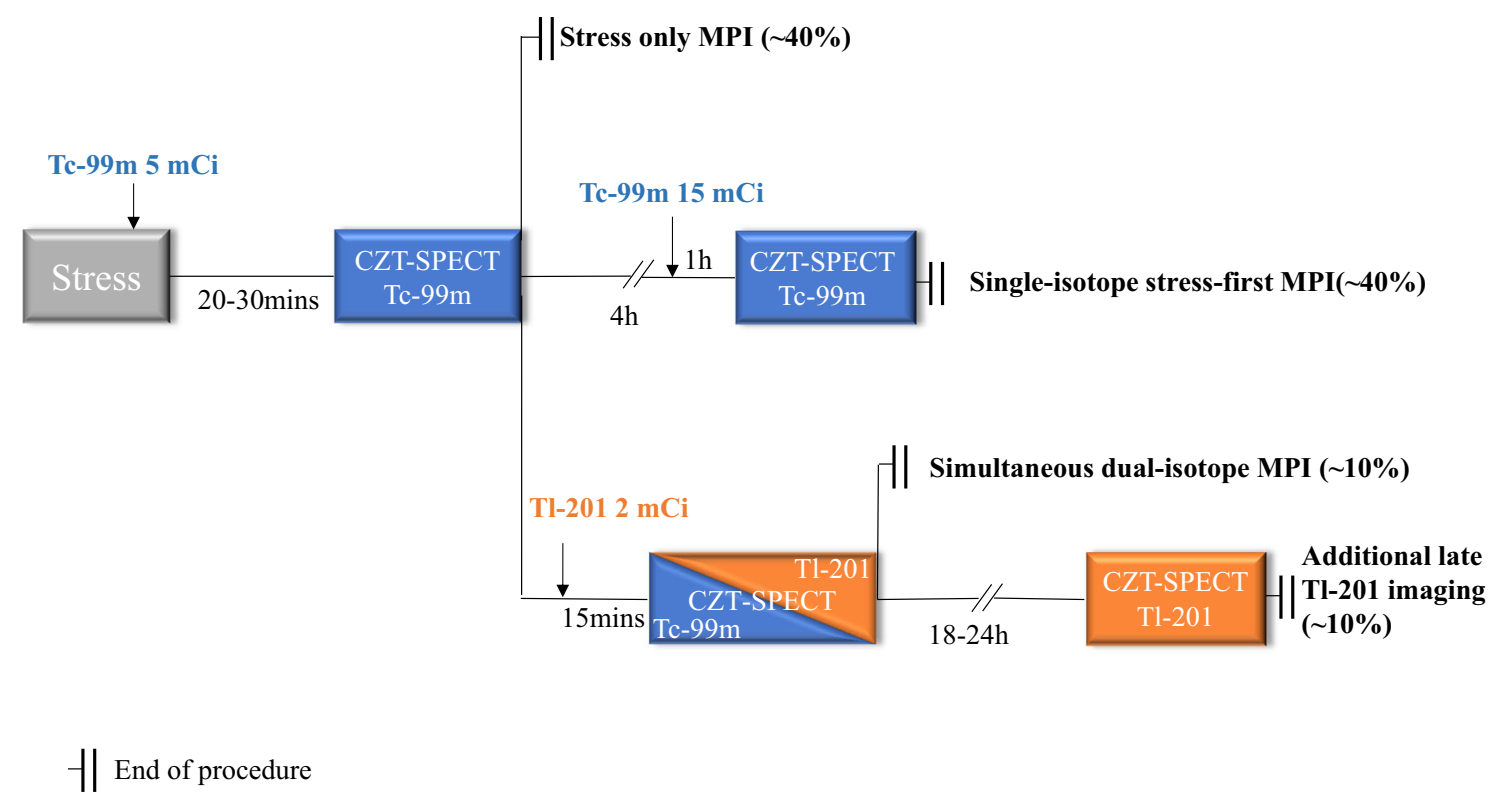

Figure 2. Incorporation of dual-isotope CZT-MPI in patient-centered nuclear cardiology program.

for assessing myocardial viability within large infarcted zones, and within areas with severe resting hypoperfusion. Single-isotope Tc-99m procedures are advantages with respect to dosimetry, protocol flexibility, and image quality. However, evaluation of patients with large infarcted myocardium and reduced ejection fraction is incomplete using these protocols. Using CZT detectors, image quality of $\mathrm{Tl}-201$ has been greatly improved compared to conventional $\mathrm{NaI}$ detectors, and the injected dose can be reduced. ${ }^{12}$ Moreover, delayed Tl-201 images 18-24 hours after injection, which suffer from poor quality using conventional SPECT are of excellent quality using CZT detectors. Thus, rest-redistribution Tl-201 as part of the dual-isotope protocol using a CZT camera might be more accurate and useful in detecting myocardial viability than could be previously obtained with conventional SPECT. Yet, because of the higher effective radiation compared to the single-isotope Tc$99 \mathrm{~m}$ procedures, this protocol should be reserved for patients with moderate or large infarcts and reduced ventricular function.

A reasonable program of a nuclear lab using a CZT camera, based on patient-centered imaging and incorporating dual-isotope MPI is demonstrated in Figure 2. Starting with a low-dose stress Tc-99m (either sestamibi or tetrofosmin) acquisition, around $40 \%$ of the studies could be converted to stress-only testing, sparing the need for a resting injection and receiving only 1-2 $\mathrm{mSv}$ effective radiation dose. Another $40 \%$ of the patients receive also a resting Tc-99m dose, with a total stress and rest radiation dose of $4.5-7 \mathrm{mSv}$. An estimate of $20 \%$ of the patients, with history of myocardial infarction and moderate-severe stress perfusion deficit at the initial singleisotope stress imaging, receive a reduced dose of T1-201 and simultaneous dual-isotope acquisition performed after 15 minutes. Those with large non-reversible or partially reversible perfusion deficit, estimated as $\sim 10 \%$ of all studies undergo a late, 18-24 hours Tl-201 imaging for the assessment of myocardial viability. In cases with known intermediate-large infarcts and left ventricular dysfunction, who are specifically referred for viability assessment, rest Tl-201 might be administered directly following stress testing, skipping the initial single-isotope stress Tc-99m acquisition. In these cases, simultaneous acquisition of stress Tc-99m and rest Tl-201 is the first acquisition performed, followed by delayed Tl-201 redistribution acquisition after 18-24 hours. Patient-centered imaging in nuclear cardiology, assessing ischemia, infarct, and viability will improve diagnosis and patient management, reduce radiation exposure, and aid in increasing patient throughput and effective utilization of nuclear lab facilities.

\section{Disclosure}

Tali Sharir has no conflict of interest. Dr. Piotr Slomka participates in software royalties at Cedars-Sinai Medical Center. 


\section{References}

1. Kawana M, Kiuzinc H, Porter J, Lathrop KA, Charleston D, Harper PV, et al. Use of TI as a potassium analog in scanning. J Nucl Med. 1970;11:333-6.

2. Lebowitz E, Greene MW, Fairchild R, Bradley-Moore PR, Atkins HL, Ansari AN, et al. Thallium-201 for medical use. I. J Nucl Med. 1975;16:151-5.

3. Berman DS, Kiat HS, Van Train KF, Germano G, Maddahi J, Friedman JD. Myocardial perfusion imaging with technetium99m-sestamibi: Comparative analysis of available imaging protocols. J Nucl Med. 1994;35:681-8.

4. Berman DS, Kiat H, Friedman JD, Wang FP, van Train K, Matzer L, et al. Separate acquisition rest thallium-201/stress technetium$99 \mathrm{~m}$ sestamibi dual-isotope myocardial perfusion single-photon emission computed tomography: A clinical validation study. J Am Coll Cardiol. 1993;22:1455-64.

5. Kiat H, Germano G, Friedman J, Van Train K, Silagan G, Wang FP, et al. Comparative feasibility of separate or simultaneous rest thallium-201/stress technetium-99 m-sestamibi dual-isotope myocardial perfusion SPECT. J Nucl Med. 1994;35:542-8.

6. Einstein AJ, Pascual TN, Mercuri M, Karthikeyan G, Vitola JV, Mahmarian JJ, et al. INCAPS Investigators Group. Current worldwide nuclear cardiology practices and radiation exposure: Results from the 65 country IAEA Nuclear Cardiology Protocols Cross-Sectional Study (INCAPS). Eur Heart J. 2015;36:1689-96.
7. Kacperski K, Erlandsson K, Ben-Haim S, Hutton BF. Iterative deconvolution of simultaneous $99 \mathrm{mTc}$ and $201 \mathrm{Tl}$ projection data measured on a CdZnTe-based cardiac SPECT scanner. Phys Med Biol. 2011;56:1397-414.

8. Gowd BM, Heller GV, Parker MW. Stress-only SPECT myocardial perfusion imaging: A review. J Nucl Cardiol. 2014;21:120012.

9. Ben-Haim S, Kacperski K, Hain S, Van Gramberg D, Hutton BF, Erlandsson K, et al. Simultaneous dual-radionuclide myocardial perfusion imaging with a solid-state dedicated cardiac camera. Eur J Nucl Med Mol Imaging. 2010;37:1710-21.

10. Berman DS, Kang X, Tamarappoo B, Wolak A, Hayes SW, Nakazato R, et al. Stress thallium-201/rest technetium-99m sequential dual isotope high-speed myocardial perfusion imaging. JACC Cardiovasc Imaging. 2009;2:273-82.

11. Makita A, Matsumoto N, Suzuki Y, Hori Y, Kuronuma K, Yoda S, et al. Clinical feasibility of simultaneous acquisition rest $(99 \mathrm{~m}) \mathrm{Tc} /$ stress (201)Tl dual-isotope myocardial perfusion single-photon emission computed tomography with semiconductor camera. Circ J. 2016;80:689-95.

12. Songy B, Lussato D, Guernou M, Queneau M, Geronazzo R. Comparison of myocardial perfusion imaging using thallium-201 between a new cadmium-zinc-telluride cardiac camera and a conventional SPECT camera. Clin Nucl Med. 2011;36:776-80. 